\title{
NanoNewton Force Sensing and Control in Microrobotic Cell Manipulation
}

\author{
Xinyu Liu, Keekyoung Kim, Yong Zhang, and Yu Sun* \\ Advanced Micro and Nanosystems Laboratory \\ University of Toronto, Canada \\ *sun@mie.utoronto.ca
}

\begin{abstract}
Cellular force sensing and control techniques are capable of enhancing the dexterity and reliability of microrobotic cell manipulation systems. This paper presents a vision-based cellular force sensing technique using a microfabricated elastic cell holding device and a sub-pixel visual tracking algorithm for resolving forces down to $3.7 \mathrm{nN}$ during microrobotic mouse embryo injection. The technique also experimentally proves useful for in situ differentiation of healthy mouse embryos from those with compromised developmental competence without the requirement of a separate mechanical characterization process. Concerning force-controlled microrobotic cell manipulation (pick-transport-place), this paper presents the first demonstration of nanoNewton force-controlled cell micrograsping using a MEMS-based microgripper with integrated two-axis force feedback. On-chip force sensors are used for detecting contact between the microgripper and cells to be manipulated (resolution: $38.5 \mathrm{nN}$ ) and sensing gripping forces (resolution: $19.9 \mathrm{nN}$ ) during force-controlled grasping. The experimental results demonstrate that the microgripper and the control system are capable of rapid contact detection and reliable force-controlled micrograsping to accommodate variations in size and stiffness of cells with a high reproducibility.
\end{abstract}

\section{INTRODUCTION}

Manipulation of single living cells represents an enabling technology that is important for a range of biological disciplines (e.g., genetics [1][2], in vitro fertilization [3], cell mechanical characterization [4], and single cell-based sensing [5]). The past decade has witnessed significant progress in the development of robotic systems and tools for conducting complex cell manipulation tasks, such as probing, characterizing, grasping, and injecting single cells.

Robotic cell manipulation is universally conducted under an optical microscope; thus, visual feedback is the main sensing modality in all existing microrobotic cell manipulation systems. Meanwhile, due to the fact that biological cells are delicate and highly deformable, quantification of interaction forces between the end-effector and cells can enhance the capability of a robotic cell manipulation system. For example, cellular force feedback was demonstrated to be useful for the alignment between a probe and a cell [4]. The measurement of cellular forces also enables the prediction of cell membrane penetration in the injection of zebrafish embryos [6][7][8].

In order to obtain cellular force feedback during microrobotic cell manipulation, the development of force sensing devices has been a focus, resulting in capacitive force sensors [4] and piezoelectric force sensors [6][9], to name just a few. Inherent limitations prevent their use in practical cell manipulation tasks: (1) these force sensors are typically limited to resolving forces at the microNewton level while the manipulation of most cell lines requires a resolution of nanoNewton or sub-nanoNewton; (2) the integration of an endeffector (e.g., glass micropipette) and the force sensors is via epoxy glue, complicating the task of end-effector exchange.

Overcoming limitations of existing cellular force sensing approaches, this paper presents a vision-based cellular force measurement technique with a nanoNewton force resolution employing a microfabricated elastic cell holding device and a sub-pixel visual tracking algorithm. The technique allows for accurately resolving cellular forces during microrobotic cell manipulation without disturbing the manipulation process or imposing difficulties in end-effector exchange. The effectiveness of the technique is demonstrated in microrobotic mouse embryo injection. Furthermore, the force sensing technique proves useful for in situ distinguishing normal embryos from those with compromised developmental competence, without requiring a separate cell characterization process.

On the front of cellular force sensing and control, the paper also presents the first demonstration of force-controlled micrograsping of biological cells at the nanoNewton force level. As mechanical end-effectors, microgrippers enable picktransport-place of biological cells in an aqueous environment. The microrobotic system employs a novel microgripper that integrates two-axis force sensors for resolving both gripping forces and contact forces between the gripping arm tips and a sample/substrate. The force-controlled microrobotic system experimentally demonstrated the capability of rapid contact detection and reliable force-controlled micrograsping of interstitial cells to accommodate variations in sizes and mechanical properties of cells with a high reproducibility.

\section{Vision-Based Cellular Force Measurement DURING CELl INJECTION}

Vision-based force measurement techniques are capable of retrieving both vision and force information from a single vision sensor (CCD/CMOS camera) under microscopic environments [10][11]. For cellular force sensing during microrobotic cell manipulation, this concept is realized by visually tracking flexible structural deformations, and subsequently, transforming material deformations into forces. 


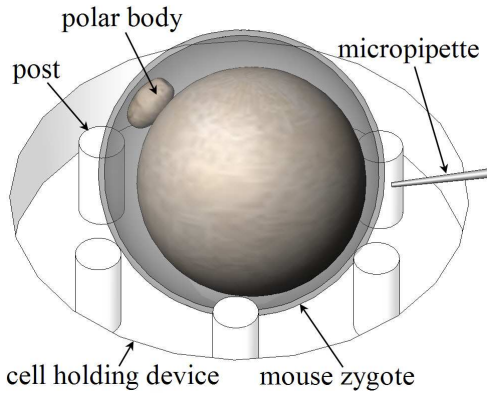

Fig. 1. Cellular force measurement using low-stiffness elastic posts during microrobotic cell injection.

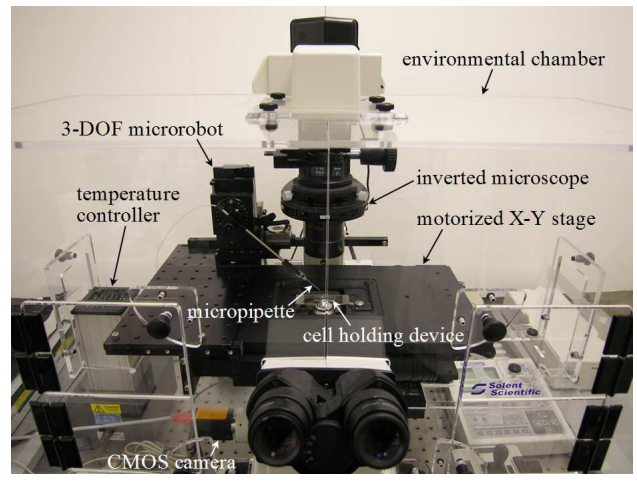

Fig. 2. Microrobotic mouse embryo injection system.

Fig. 1 schematically illustrates the principle of vision-based cellular force measurement using an elastic cell holding device during microrobotic cell injection. While the micropipette injects individual cells inside cavities on a cell holding device, applied forces are transmitted to low-stiffness, supporting posts. In real time, a sub-pixel visual tracking algorithm measures post deflections that are fitted into an analytical mechanics model to calculate the force exerted on the cell.

This technique was previously demonstrated on zebrafish embryos [12]. The study presented in this paper focuses on investigating the feasibility of further miniaturizing the cell holding devices to accommodate mouse embryos $(100 \mu \mathrm{m}$ in diameter vs. $1.3 \mathrm{~mm}$ zebrafish embryos) for measuring nanoNewton cellular forces during microinjection; and the possibility of using cellular force information to distinguish normal mouse embryos from those with compromised developmental competence for better selecting healthy embryos in genetics and reproductive research.

\section{A. Microrobotic Mouse Embryo Injection System}

The microrobotic mouse embryo injection system (Fig. 2) consists of a polydimethylsiloxane (PDMS) cell holding device, an inverted microscope (TE2000, Nikon) with a CMOS digital camera (A601f, Basler), a 3-DOF microrobot (MP-285, Sutter) for controlling the micropipette motion, a motorized XY stage (ProScan II, Prior Scientific) for positioning cell samples, and a temperature-controlled chamber (Solent Scientific) to maintain cells at $37^{\circ} \mathrm{C}$.

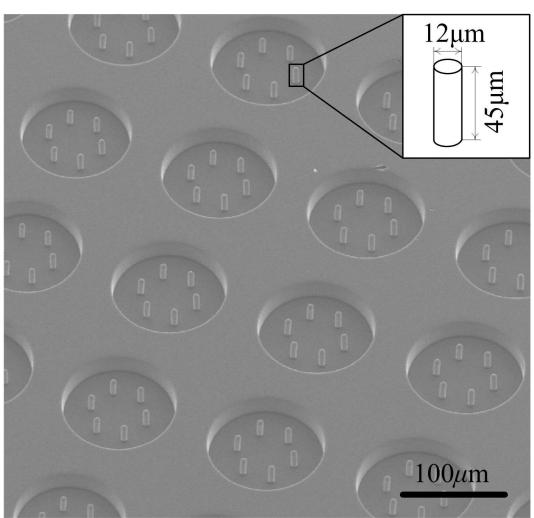

Fig. 3. SEM image of a PDMS cell holding device.

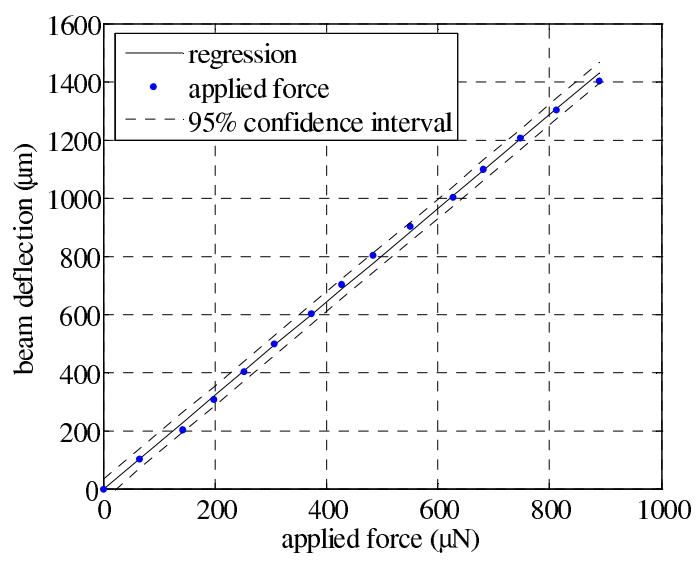

Fig. 4. Young's modulus calibration on a bulkier PDMS beam.

\section{B. Fabrication and Characterization of Cell Holding Devices}

The cell holding device shown in Fig. 3 was constructed with PDMS via soft lithography [12]. Briefly, PDMS prepolymer prepared by mixing Sylgard 184 (Dow Corning) and its curing agent with a weight ratio of $15: 1$, was poured over a SU-8 mold (SU-8 50, MicroChem) made on a silicon wafter using standard photolithography. After curing at $80^{\circ} \mathrm{C}$ for $8 \mathrm{hr}$, the PDMS devices were peeled off the SU-8 mold. The depth of the cavity and protruding posts is $45 \mu \mathrm{m}$, and the diameter of the posts is $12 \mu \mathrm{m}$ (Fig. 3). In order to make the PDMS surface hydrophilic, the devices were oxygen plasma treated for $10 \mathrm{sec}$ before use.

To determine the Young's modulus of the cell holding device, a bulkier PDMS beam produced under exactly the same processing conditions was calibrated with a piezoresistive force sensor (AE801, SensorOne) as described in [12]. It has been demonstrated that the Young's modulus values characterized from bulk PDMS and a micro PDMS structure, both constructed with the same microfabrication parameters, differ within 5\% [13]. Fig. 4 shows the calibration data of applied force vs. beam deflection. The determined Young's modulus value is $422.4 \mathrm{kPa}$. 


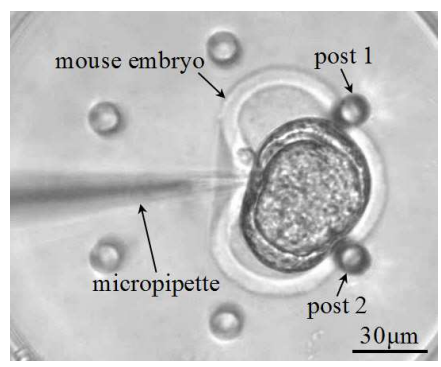

Fig. 5. Indentation forces deform the mouse embryo and deflect two supporting posts. (a)

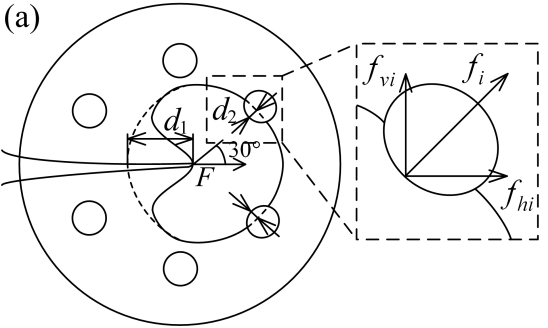

(b)

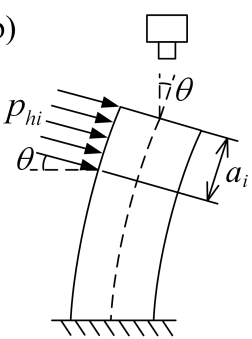

Fig. 6. Injection force analysis. (a) Force balance on the cell under indentation. (b) Post deflection model.

\section{Mouse Embryo Preparation}

As a model organism, mouse is a primary animal for genetics and reproductive research. Besides the importance in in vitro fertilization, microinjection of mouse oocytes and embryos is important for screening molecular targets linked to the study of basic biology of embryo development, such as mitochondrial-associated recombinant proteins, neutralizing antibody, morpholinos, and expression vectors for siRNA.

The mouse embryos used in this research were collected according to standard protocols approved by the Mount Sinai Hospital Animal Care Committee in Toronto. Young (8-12 weeks old) and older (40 weeks old) ICR female mice were used for obtaining normal embryos and those with blastomere fragmentation. ICR females with different ages were superovulated by injecting equine pregnant mare's serum gonadotropin (PMSG) and human chorionic gonadotropin (hCG) 48hr later. The mice were subsequently mated with ICR males of proven fertility, and plugs were verified the next morning. In vivo fertilized embryos were collected from the mated female mice at $24 \mathrm{hr}$ post-hCG and cultured in human tubal fluid (HTF) to two-cell stage (at $48 \mathrm{hr}$ post-hCG). The average diameter of the mouse embryos is $98 \mu \mathrm{m}$.

\section{Force Analysis}

Fig. 5 shows a snapshot captured in the cell injection process. The microrobot controls an injection micropipette to exert an indentation force to a mouse embryo, deflecting the two supporting posts on the opposite side. Post deflections, measured by a visual tracking algorithm that will be discussed in Section II-E, are fitted to an analytical mechanics model to obtain contact forces between the cell and posts. Based on the contact forces, the indentation force applied by the micropipette on the cell is determined through the following force analysis.

The cell is treated as elastic due to the fact that quick indentation by the micropipette does not leave sufficient time for cellular creep or relaxation to occur. Consequently, the injection force, $F$ is balanced by the horizontal components, $f_{h i}$ of contact forces between the cell and supporting posts (Fig. 6(a)),

$$
F=\sum_{i=1}^{2} f_{h i}
$$

Much higher deformability of mouse embryos than that of zebrafish embryos results in different contact behavior between a cell and supporting posts, necessitating different treatments of forces in analysis. In the device configuration, the radius of the cell $(\sim 49 \mu \mathrm{m})$ is larger than the depth of the cavity and posts $(45 \mu \mathrm{m})$, resulting in an initial point contact between the cell and supporting posts before post deflections occur. However, the high deformability of mouse embryos makes cell membrane conform to the posts when an injection force is applied to the cell. It is assumed that the contact forces are evenly distributed over the contact areas. Thus, the horizontal components, $f_{h i}$ are expressed by a constant force intensity, $p_{h i}$ and a contact length, $a_{i}$ (Fig. 6(b))

$$
f_{h i}=p_{h i} a_{i}
$$

Slope $\theta$ of the posts' free ends shown in Fig. 6(b) was measured to verify the validity of linear elasticity that requires small structural deflections. The maximum slope was determined to be $11.1^{\circ}$, which satisfies $\sin \theta \approx \theta$; thus, the small deflection assumption of linear elasticity holds [14]. Therefore, the relationship of the horizontal force intensity, $p_{h i}$ and post deflections can be established [14].

$$
p_{h i}=\frac{\delta_{i}}{\frac{40 a_{i}(1+\gamma)\left(2 H-a_{i}\right)}{9 \pi E D^{2}}+\frac{8\left(a_{i}^{4}+8 H^{3} a_{i}-6 H^{2} a_{i}^{2}\right)}{3 \pi E D^{4}}}
$$

where $i=1,2 ; \delta_{i}$ is the horizontal deflection; $H$ and $D$ are post height and diameter; $E$ and $\gamma$ are Young's modulus and Poisson's ratio $(\gamma=0.5$ for PDMS [12]).

Combining (1)-(3) yields the injection force applied by the micropipette to the cell.

$$
F=\sum_{i=1}^{2} \frac{\delta_{i} a_{i}}{\frac{40 a_{i}(1+\gamma)\left(2 H-a_{i}\right)}{9 \pi E D^{2}}+\frac{8\left(a_{i}^{4}+8 H^{3} a_{i}-6 H^{2} a_{i}^{2}\right)}{3 \pi E D^{4}}}
$$

In (4), the unknown parameters are post horizontal deflections, $\delta_{i}$ and the contact length, $a_{i}$. Experimentally, imaging with a side-view microscope confirmed that the contact length, $a_{i}$ increases at a constant speed, $v$ for a given indentation speed. Hence, $a_{i}=v t$, where $t$ denotes time.

Note that for a constant indentation speed of the micropipette, the variation speed of $a_{i}, v$ varies for different cells. At $20 \mu \mathrm{m} / \mathrm{sec}$ used throughout the experiments, $v$ of the six tested mouse embryos were measured to be $0.8-1.2 \mu \mathrm{m} / \mathrm{sec}$. Interestingly, the sensitivity of the mechanics model (4) to variations in $v$ is low. The injection force varies only by 


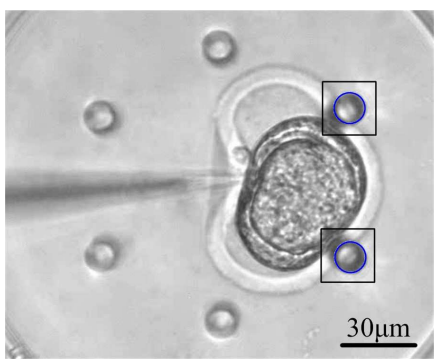

Fig. 7. Image patches tracked by template matching and LSCD detected post top circles.

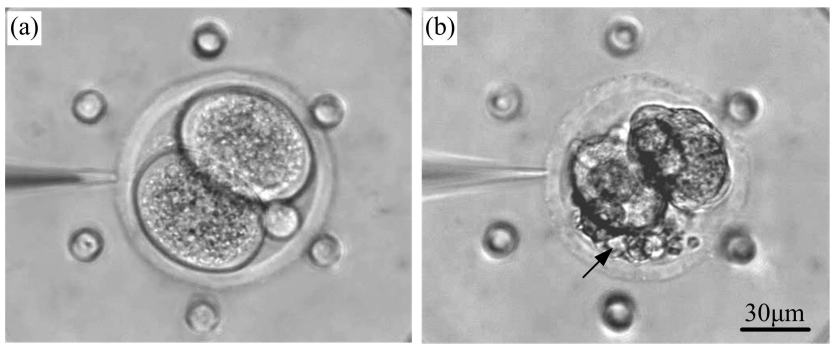

Fig. 8. Mouse embryos for cellular force measurement. (a) Normal embryo. (b) Embryo with blastomere fragmentation (arrow labeled).

$1 \%$ when $v$ changes from $0.8 \mu \mathrm{m} / \mathrm{sec}$ to $1.2 \mu \mathrm{m} / \mathrm{sec}$. Thus, the average value of the measured speeds, $1 \mu \mathrm{m} / \mathrm{sec}$ was used to calculate injection forces for all the embryos.

\section{E. Visual Tracking of Post Deflections}

In order to accurately track post deflections, a visual tracking algorithm with a resolution of 0.5 pixel was developed and described in detail in [12]. A template matching algorithm with constant template updates first tracks the motion of the supporting posts, providing processing areas for a least squares circle detection (LSCD) algorithm to determine posts' center positions. The LSCD algorithm utilizes Canny edge detector to obtain an edge image and then extracts a portion of the post top surface for circle fitting. Fig. 7 shows the tracked image patches and LSCD detected post top circles.

\section{F. Experimental Results and Discussion}

As mouse embryos are exquisitely sensitive to slight temperature variations, experiments were conducted at $37^{\circ} \mathrm{C}$ inside the temperature-controlled chamber. With a $40 \times$ objective (NA $0.55)$, the pixel size of the imaging system was calibrated to be $0.36 \mu \mathrm{m} \times 0.36 \mu \mathrm{m}$. Micropipette tips used for indenting mouse embryos was $1.8 \mu \mathrm{m}$ in diameter.

Three normal ICR embryos and three ICR embryos with blastomere fragmentation at the two-cell stage were used for cellular force measurements. Blastomere fragmentation is often indicative of future programmed cell death [15]. Although the blastomere fragmented embryo shown in Fig. 8 can be distinguished morphologically from normal embryos, using morphological differences alone is not always effective to distinguish diseased embryos from normal embryos due to the fact that one fourth of the fragmented embryos are

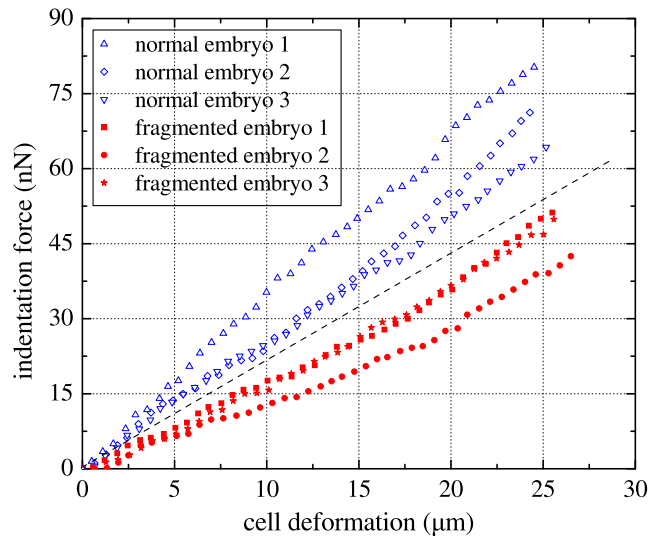

Fig. 9. Force-deformation curves of normal embryos (blue) and fragmented embryos (red). They separate themselves into two distinct regions.

TABLE I

SLOPES OF CELlular ForCe-Deformation CuRVES.

\begin{tabular}{c|c|c}
\hline mouse embryo & $\begin{array}{c}\text { slope } \\
(\mathrm{nN} / \mu \mathrm{m})\end{array}$ & $\begin{array}{c}\text { slope average } \\
(\mathrm{nN} / \mu \mathrm{m})\end{array}$ \\
\hline normal embryo 1 & 3.35 & \\
normal embryo 2 & 2.74 & $2.87 \pm 0.43$ \\
normal embryo 3 & 2.52 & \\
\hline fragmented embryo 1 & 1.84 & \\
fragmented embryo 2 & 1.45 & $1.70 \pm 0.22$ \\
fragmented embryo 3 & 1.81 & \\
\hline
\end{tabular}

able to develop normally [15]. We hypothesized that subtle changes in the cytoskeleton structure could lead to stiffness changes between abnormal and normal embryos. Thus, cellular force-deformation measurements were expected to provide additional information for detecting embryonic dysfunctions that require assisted hatching and for helping better select healthy embryos for implantation after microinjection.

The six embryos were manually delivered onto the cell holding device using a transfer pipette and then indented via microrobotic teleoperation. The micropipette was controlled to indent each embryo by $30 \mu \mathrm{m}$ at the speed of $20 \mu \mathrm{m} / \mathrm{sec}$. During the indentation process, force data were collected in real time (30 data points per sec).

Fig. 9 shows force-deformation curves of both normal and fragmented embryos. The horizontal axis represents cell deformation, $d=d_{1}+d_{2} \cos 30^{\circ}$, where $d_{1}$ and $d_{2}$ were defined in Fig. 6. The vertical axis shows vision-based cellular force data. With the current cell holding devices and imaging system, the force measurement resolution was determined to be $3.7 \mathrm{nN}$.

From Fig. 9, it can be seen that the force-deformation curves of normal and fragmented embryos separate themselves into two distinct regions. Table I summarizes the curve slopes calculated by linear regression. The slopes for normal embryos range from $2.52 \mathrm{nN} / \mu \mathrm{m}$ to $3.35 \mathrm{nN} / \mu \mathrm{m}$ while the slopes for fragmented embryos are between $1.45 \mathrm{nN} / \mu \mathrm{m}$ and $1.84 \mathrm{nN} / \mu \mathrm{m}$, quantitatively demonstrating that the normal embryos and fragmented embryos are mechanically different.

All the indented embryos were subsequently cultured in an incubator at $37^{\circ} \mathrm{C}$ with $5 \% \mathrm{CO}_{2}$. The three normal embryos successfully developed into the four-cell stage; however, the 


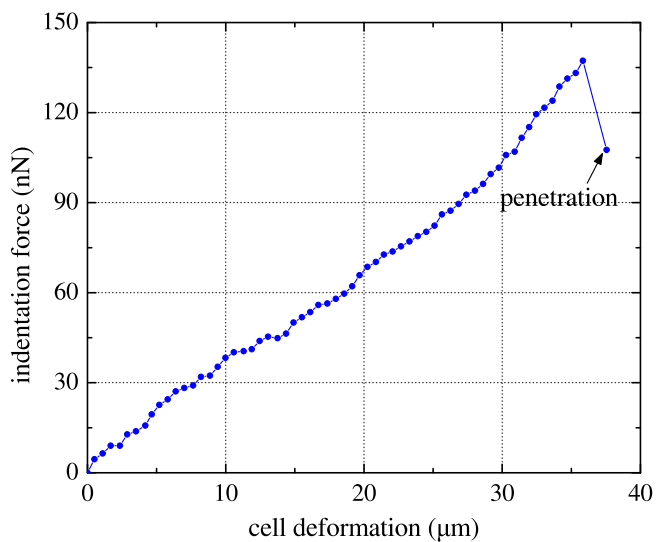

Fig. 10. Abrupt force change indicates cell membrane penetration during microrobotic cell injection.

three fragmented embryos were arrested at the two-cell stage, proving that the cellular force measurement results could be useful for distinguishing normal embryos from those with embryonic defects during microrobotic cell injection without a separate cell characterization process.

In addition, the cellular force sensing technique can also be used for detecting the penetration of cell membrane in microrobotic cell injection. An abrupt change of cellular forces (Fig. 10) indicates cell membrane penetration for subsequent material deposition. The cellular force does not return to the zero level immediately after penetration since the indented cell does not have sufficient time for recovery during injection. The force required to penetrate the outside membrane (zona pellucida) of a healthy ICR embryo was $137.3 \mathrm{nN}$.

\section{NANONEWTON ForCe-CONTROLled Micrograsping OF Biological CELLS}

Compared with end-effectors with a single tip such as a probe or a micropipette for microrobotic cell manipulation, a microgripper with two gripping arms is a more powerful tool for reliable pick-transport-place tasks. Concerning force sensing and control in microgripper-based microrobotic cell manipulation, this section presents the first demonstration of force-controlled micrograsping of biological cells at the nanoNewton force level, which was conducted with a novel, monolithic MEMS-based microgripper with integrated twoaxis force sensors.

\section{A. Microrobotic System for Force-Controlled Micrograsping}

1) System Setup: The microrobotic system shown in Fig. 11 includes a 3-DOF microrobot (MP-285, Sutter) for positioning the microgripper, a motorized X-Y stage (ProScan II, Prior Scientific) for positioning cell samples, an inverted microscope (TE2000, Nikon) with a CMOS camera (A601f, Basler), a microgripper wire bonded on a circuit board, and a motion control board (6259, National Instruments) mounted on a host computer. The microgripper was tilted at an angle of $40^{\circ}$ to enable the gripping arm tips to reach samples on the substrate without immersing the actuator or force sensors into the culture medium.

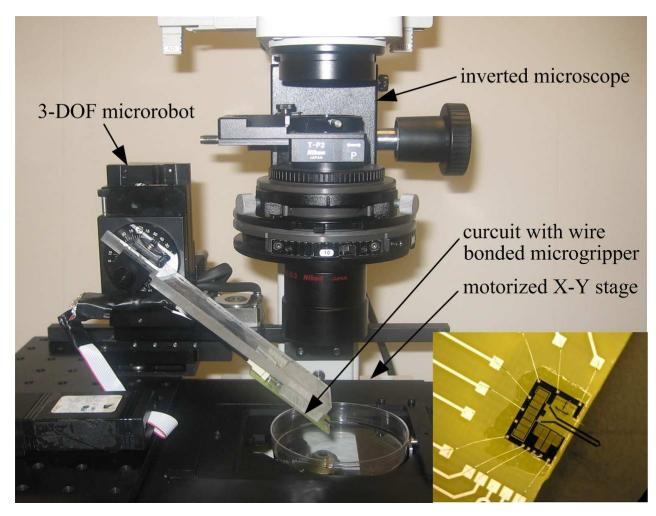

Fig. 11. Microrobotic system setup for Force-controlled micrograsping. Inlet picture shows the wire-bonded microgripper.

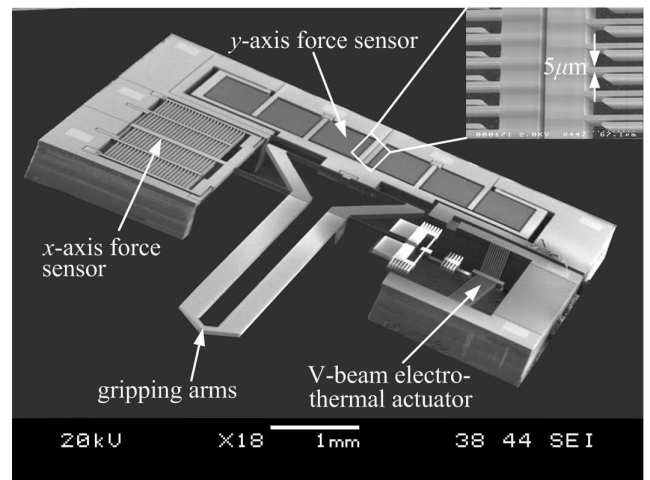

Fig. 12. MEMS-based microgripper with integrated two-axis capacitive force sensors.

2) MEMS Microgripper: Over the past two decades, many MEMS microgrippers were developed using different mechanical structures and actuation principles. For example, electrothermal microgrippers without force feedback were developed for cell manipulation [16] and carbon nanotube grasping [17]. Electrostatic microgrippers with a single-axis force sensor was reported for open-loop micrograsping [18] and for investigating charge transport through DNA [19]. Forcecontrolled micro and nanomanipulation requires microgrippers ideally capable of providing multi-axis force feedback: to protect the fragile microgripper by detecting contact between the microgripper and object to be manipulated; and to provide gripping force feedback for achieving secured grasping without applying excessive forces.

The MEMS microgripper with integrated two-axis force sensors, shown in Fig. 12 was constructed through a modified DRIE-SOI process. The device employs a V-beam electrothermal actuator that is connected to the lower part of a long gripping arm to generate large gripping displacements at gripping arm tips with low driving voltages. As shown in Fig. 13, the gripping arm tip moves by $32 \mu \mathrm{m}$ at $6 \mathrm{~V}$; and due to the many heat sink beams, the measured temperature at the gripping arm tip is $29^{\circ} \mathrm{C}$ in air, demonstrating a low temperature suitable for biomaterial manipulation.

Integrated two-axis capacitive force sensors are imple- 


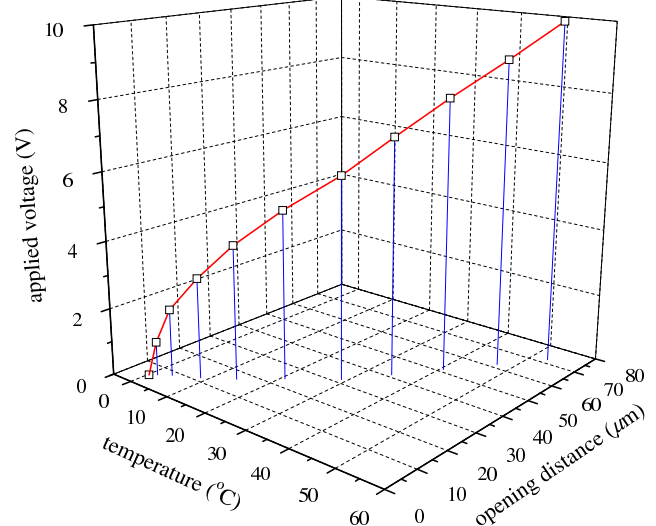

Fig. 13. Measured gripping arm tip displacement and temperature at actuation voltages of $1-10 \mathrm{~V}$.

(a)

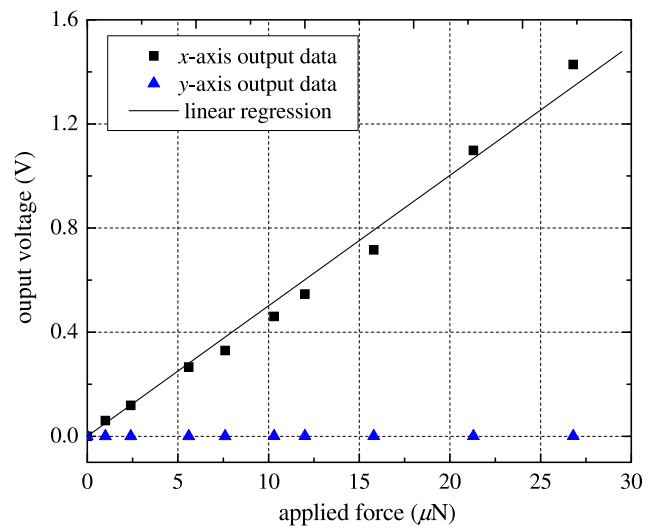

(b)

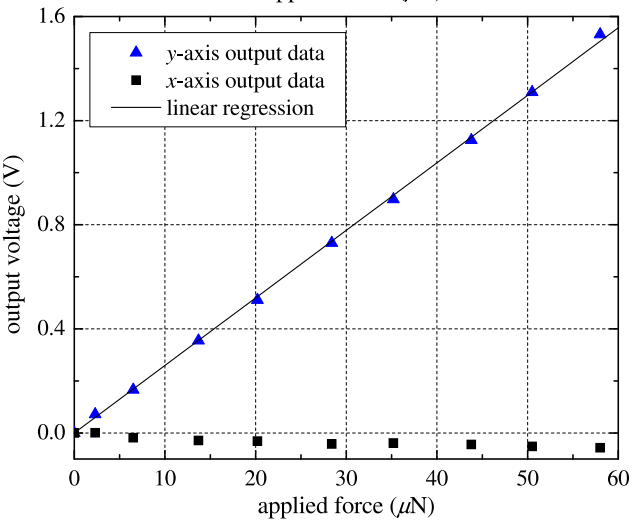

Fig. 14. Force sensor calibration results. Forces applied only (a) along the $x$ direction; (b) only along the $y$ direction. Also shown are coupled responses.

mented with transverse differential comb drives and are orthogonally configured. The contact force feedback (ydirectional) enables contact detection and protection of the microgripper from breakage. The gripping force feedback ( $x$-directional) permits force-controlled micrograsping with a force controller to accommodate size and stiffness variations of objects to achieve secured grasping with no excessive forces applied. Fig. 14 shows the force sensor calibration results, demonstrating a high input-output linearity and minimized cross-axis coupling. The integrated force sensors are capable of resolving gripping forces up to $30 \mu \mathrm{N}$ (resolution: $19.9 \mathrm{nN}$ ) and contact forces up to $58 \mu \mathrm{N}$ (resolution: $38.5 \mathrm{nN}$ ).

\section{B. Interstitial Cell Preparation}

Porcine aortic valve interstitial cells (PAVICs) were manipulated to demonstrate force-controlled micrograsping. Manipulation of single PAVICs with cellular force feedback is required for cell transfer and mechanical characterization in pharmacological studies, such as heart aortic valve calcification.

Aortic valve leaflets were harvested from healthy pig hearts obtained at a local abattoir. After rinsing with antibiotics, each leaflet was treated with collagenase $\left(150 \mathrm{U} / \mathrm{mL}, 37^{\circ} \mathrm{C}\right.$, $20 \mathrm{~min}$ ) and the leaflet surfaces were scraped to remove endothelial cells. The leaflets were then minced, and digested with collagenase $\left(150 \mathrm{U} / \mathrm{mL}, 37^{\circ} \mathrm{C}, 2 \mathrm{hr}\right)$. The interstitial cells were enzymatically isolated, grown on tissue culture flasks, and kept in an incubator in standard tissue culture medium (DMEM supplemented with 10\% FBS and 1\% antibiotics). The medium was changed every 2 days, and the cells were passaged when confluent. P2 cells were trypsinized and resuspended in standard tissue culture medium at $10^{5}$ cells $/ \mathrm{mL}$ for use in experiments.

\section{Experimental Results and Discussion}

The experiments were conducted at room temperature $\left(23^{\circ} \mathrm{C}\right)$. In order to reduce adhesion of cells to the gripping arm tips and thus, facilitate cell release, the microgripper tips were dip coated with $10 \%$ SurfaSil siliconizing fluid (Pierce Chemicals) and $90 \%$ histological-grade xylenes (Sigma-Aldrich) for $10 \mathrm{sec}$ before use.

1) Contact Detection: A droplet of cell culture medium containing suspended PAVICs $(10-20 \mu \mathrm{m}$ in diameter) was dispensed through pipetting on a polystyrene petri dish. After PAVICs settle down on the substrate, a microrobot controls the microgripper to immerse gripping arm tips into the liquid droplet and conducts contact detection.

Contact detection is important to protect the microgripper from damage. After the tips of gripping arms are immersed into the medium, the microrobot controls the microgripper at a constant speed of $20 \mu \mathrm{m} / \mathrm{sec}$ to approach the substrate while force data along the y direction are sampled. The contact detection process completes within $5 \mathrm{sec}$. Without the integrated contact force sensor, this process would be extremely time consuming and operator skill dependent.

When the monitored contact force level reaches a pre-set threshold value, it indicates that contact between the gripping arm tips and the substrate is established. Subsequently, the microrobot stops lowering the microgripper further and moves the microgripper upwards until the contact force returns to zero (Fig. 15). After the initial contact position is detected, the microgripper is positioned a few micrometers above the detected contact position. The pre-set threshold force value used in the experiments was $150 \mathrm{nN}$, which was effective for reliably determining the initial contact between the gripping arm tips and the substrate. 


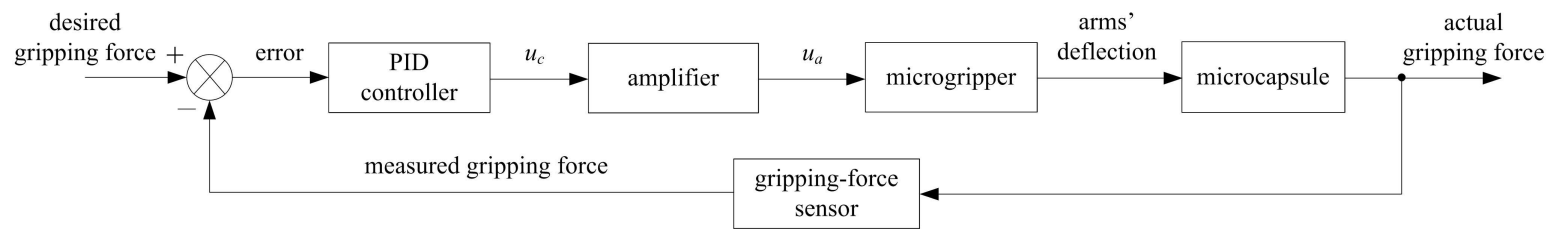

Fig. 17. Block diagram of force-controlled micrograsping.

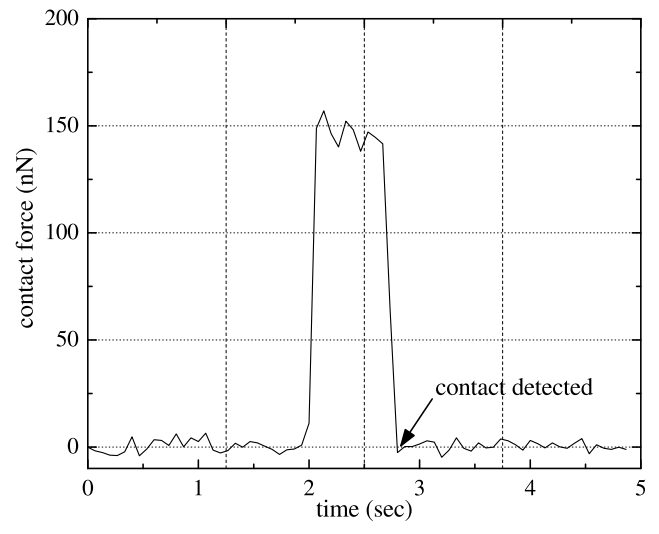

Fig. 15. Contact force monitoring for reliable contact detection.

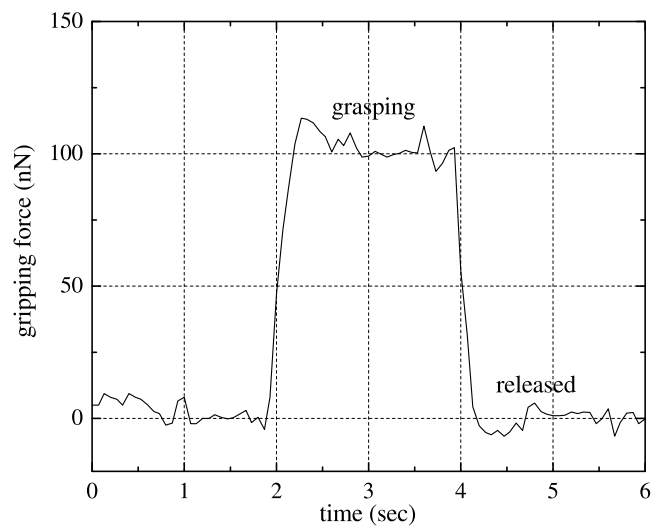

Fig. 16. Gripping force profile during micrograsping and releasing of a cell.

2) Force-Controlled Grasping of Biological Cells: Before the system performed force-controlled micrograsping of PAVICs, experiments were conducted to evaluate the effectiveness of open-loop micrograsping. The system applies a voltage to the V-beam electrothermal actuator to produce an opening larger than the size of a PAVIC between the two gripping arms. When grasping a target PAVIC, the system reduces the applied voltage level, which decreases the arm opening and realizes grasping.

Fig. 16 shows the force profile during cell grasping and releasing, where a sequence of actuation voltages was applied $(5 \mathrm{~V}$ opening voltage, $1.5 \mathrm{~V}$ grasping voltage, and $5 \mathrm{~V}$ releasing voltage) to grasp and release a $15 \mu \mathrm{m}$ PAVIC. At $1.5 \mathrm{~V}$ grasping voltage, the PAVIC was experiencing a gripping force of $100 \mathrm{nN}$ that produced $15 \%$ cell deformation of its diameter. Due to different sizes of PAVICs and their stiffness variations, a single fixed grasping voltage can often cause

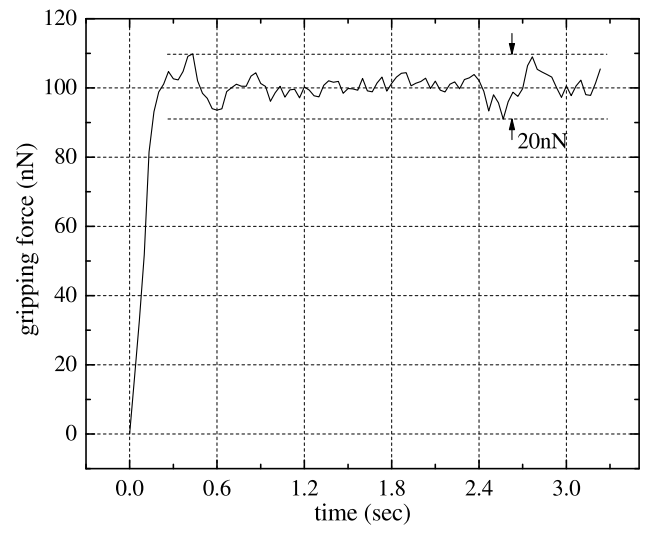

Fig. 18. Step response of force-controlled micrograsping.

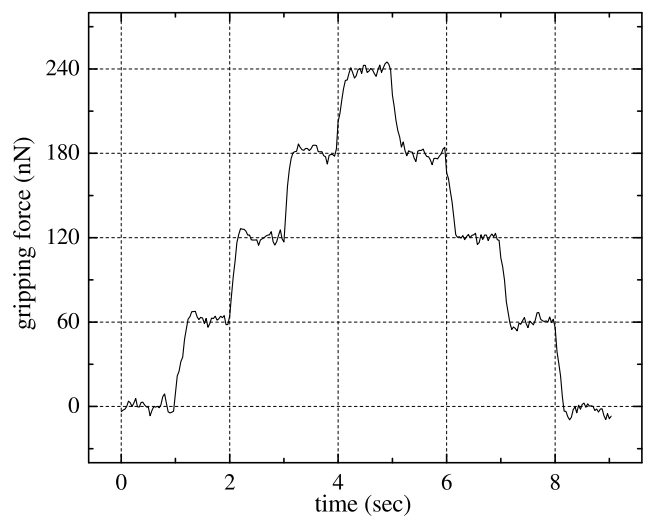

Fig. 19. Tracking force steps with an increment of $60 \mathrm{nN}$.

either unsecured grasping or cell rupturing from excessively applied forces, necessitating closed-loop force-controlled micrograsping.

To achieve reliable micrograsping, a closed-loop control system was implemented by using gripping force signals as feedback to form a closed loop. Fig. 17 shows the block diagram of the force control system that accepts a pre-set force level as reference input and employs proportional-integralderivative (PID) control for force-controlled micrograsping. Fig. 18 shows the step response of the force-controlled micrograsping system to track a reference input of $100 \mathrm{nN}$. The settling time is approximately $200 \mathrm{~ms}$. Corresponding to reference input force steps with an increment of $60 \mathrm{nN}$, tracking results are shown in Fig. 19.

Enabled by the monolithic microgripper with two-axis force feedback, the microrobotic system demonstrates the capability of rapidly detecting contact, accurately tracking nanoNew- 

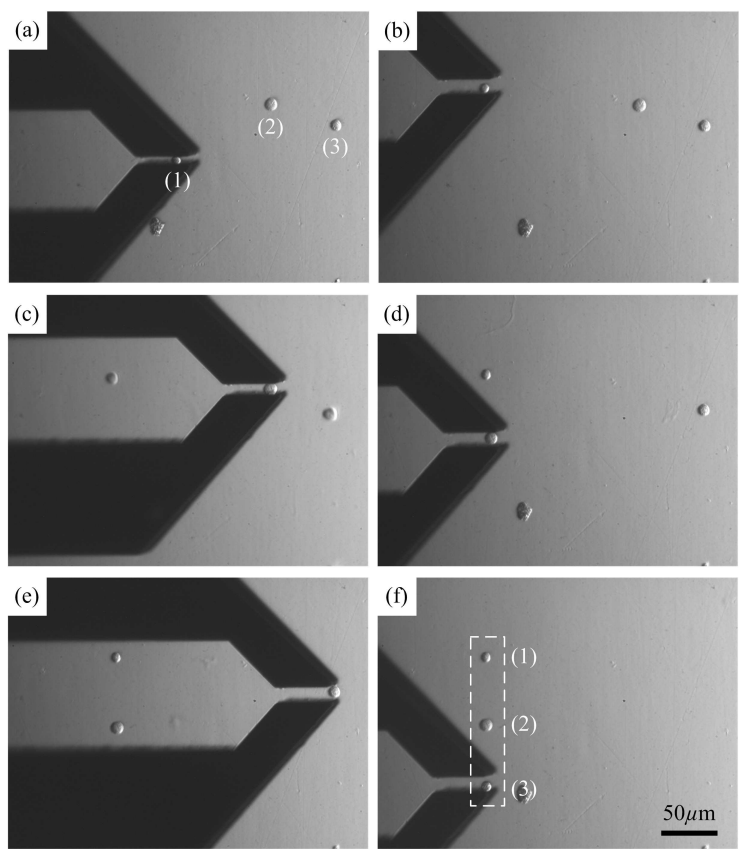

Fig. 20. Cell manipulation and alignment with force-controlled micrograsping. (a) After contact detection, the microgripper grasps a first cell. (b) The microgripper transfers the cell to a new position and releases the cell. (c) The microgripper grasps a second cell. (d) Transferring and releasing the second cell. (e) The microgripper approaches a third cell. (f) Transferring and releasing the third cell. Three cells of different sizes are transferred to desired positions and aligned.

ton gripping forces, and performing reliable force-controlled micrograsping to accommodate size and mechanical property variations of objects. Fig. 20 shows three PAVICs of different sizes that were picked, placed, and aligned. Force-controlled micrograsping of the aligned PAVICs was conducted at a force level of $65 \mathrm{nN}$.

\section{CONCLUSION}

This paper presented nanoNewton cellular force sensing and control in microrobotic cell manipulation. A vision-based cellular force sensing technique was demonstrated for resolving forces with a resolution of $3.7 \mathrm{nN}$ during microrobotic mouse embryo injection, based on a microfabricated PDMS cell holding device and a sub-pixel computer vision algorithm with a 0.5 pixel resolution. The acquired cellular force-deformation curves provided important information for differentiating normally developed mouse embryos from those with compromised developmental competence. Additionally, nanoNewton force-controlled micrograsping of cells was demonstrated. The microrobotic system used a close-loop force controller to control a MEMS-based microgripper with integrated two-axis force sensors. The contact force sensor enables rapid detection of the contact between the substrate and gripping arm tips. A PID force controller was used to regulate gripping forces for force-controlled micrograsping. Experimental results on forcecontrolled grasping of interstitial cells demonstrated that the microrobotic system is capable of performing reliable forcecontrolled manipulation at a force level of $20 \mathrm{nN}$.

\section{ACKNOWLEDGMENT}

The authors thank Jurisicova and Casper groups for the assistance with mouse embryo preparation, and Simmons group for assistance with interstitial cell preparation. This work was supported by the Natural Sciences and Engineering Research Council of Canada, Ontario Centers of Excellence, and the Ontario Ministry of Research and Innovation.

\section{REFERENCES}

[1] Y. Sun and B.J. Nelson, "Biological cell injection using an autonomous microrobotic system," Int. J. Robot. Res., Vol. 21, No. 10-11, pp. 861$868,2002$.

[2] W.H. Wang, X.Y. Liu, D. Gelinas, B. Ciruna, and Y. Sun, "A fully automated robotic system for microinjection of zebrafish embryos," PLoS ONE, Vol. 2, No. 9, e862. doi:10.1371/journal.pone.0000862, 2007.

[3] Y. Kimura and R. Yanagimachi, "Intracytoplasmic sperm injection in the mouse," Biol. Reprod., Vol. 52, pp. 709-720, 1995.

[4] Y. Sun, K.T. Wan, B.J. Nelson, J. Bischof, and K. Roberts, "Mechanical property characterization of the mouse zona pellucida," IEEE Trans. NanoBioSci., Vol. 2, No. 4, pp. 279-286, 2003.

[5] R.J. Whelan and R.N. Zare, "Single-cell immunosensors for protein detection," Biosens. Bioelectron., Vol. 19, No. 4, pp. 331-336, 2003.

[6] A. Pillarisetti, M. Pekarev, A.D. Brooks, and J.P. Desai, "Evaluating the effect of force feedback in cell injection," IEEE Trans. Autom. Sci. Eng., Vol. 4, No. 3, pp. 322-331, 2007.

[7] Z. Lu, P.C.Y. Chen, J. Nam, R. Ge, and W. Lin, "A micromanipulation system with dynamic force-feedback for automatic batch microinjection," J. Micromech. Microeng., Vol. 17, pp. 314-321, 2007.

[8] H.B. Huang, D. Sun, J.K. Mills, and W.J. Li, "Visual-based Impedance Force Control of Three-dimensional Cell Injection System,' IEEE Conf. ICRA, Rome, Italy, April 2007.

[9] D.H. Kim, C.N. Hwang, Y. Sun, S.H. Lee, B.K. Kim, and B.J. Nelson, "Mechanical analysis of chorion softening in prehatching stages of zebrafish embryos," IEEE Trans. NanoBioSci., Vol. 5, No. 2, pp. 8994, 2006.

[10] M.A. Greminger and B.J. Nelson, "Vision-based force measurement," IEEE Trans. Pattern Anal. Mach. Intell., Vol. 26, No. 3, pp. 290-298, 2004.

[11] V.V. Dobrokhotov, M.M. Yazdanpanah, S. Pabba, A. Safir, and R.W. Cohn, "Visual force sensing with flexible nanowire buckling springs," Nanotechnology, Vol. 19, No. 3, 035502, 2008.

[12] X.Y. Liu, Y. Sun, W.H. Wang, and B.M. Lansdorp, "Vision-based cellular force measurement using an elastic microfabricated device," $J$. Micromech. Microeng., Vol. 17, pp. 1281-1288, 2007.

[13] Y. Zhao, C.C. Lim, D.B. Sawyer, R. Liao, and X. Zhang, "Microchip for subcelluar mechanics study in living cells," Sens. Actuat. B - Chem., Vol. 114, pp.1108-1115, 2006.

[14] A.C. Ugural and S.K. Fenster, Advanced Strength and Applied Elasticity, Prentice Hall, 2003.

[15] A. Jurisicova, S. Varmuza, R.F. Casper, "Involvement of programmed cell death in preimplantation embryo demise," Hum. Reprod. Update, Vol. 1, pp. 558-566, 1995.

[16] N. Chronis and L.P. Lee, "Electrothermally activated SU-8 microgripper for single cell manipulation in solution," J. Microelectromech. Syst., Vol. 14, pp. 857-863, 2005.

[17] K. Carlson, K.N. Andersen, V. Eichhorn, D.H. Petersen, K. Mølhave, I.Y.Y. Bu, K.B.K. Teo, W.I. Milne, S. Fatikow, and P. Bøggild, "A carbon nanofibre scanning probe assembled using an electrothermal microgripper,", Nanotechnology, Vol. 18, 375501, 2007.

[18] F. Beyeler, A. Nield, S. Oberti, D.J. Bell, Y. Sun, J. Dual, and B.J. Nelson, "Monolithically fabricated microgripper with integratred force sensor for manipulating micro-objects and biological cells aligned in an ultrasonic field," J. Microelectromech. Syst., Vol. 16, No. 1, pp. 7-15, 2007.

[19] C. Yamahata, D. Collard, T. Takekawa, M. Kumemura, G. Hashiguchi, and H. Fujita, "Humidity dependence of charge transport through DNA revealed by silicon-based nanotweezers manipulation," Biophys. J., Vol. 94, pp. 63-70, 2008. 